\title{
Efficient spectral domain MoM for the design of circularly polarized reflectarray antennas made of split rings
}

\author{
Rafael Florencio, Rafael R. Boix.
}

\author{
and José Encinar
}

\begin{abstract}
The Method of Moments (MoM) in the spectral domain is used for the analysis of the scattering of a plane wave by a multilayered periodic structure containing conducting concentric split rings in the unit cell. Basis functions accounting for edge singularities are used in the approximation of the current density on the split rings, which makes it possible a fast convergence of $\mathrm{MoM}$ with respect to the number of basis functions. Since the 2-D Fourier transforms of the basis functions cannot be obtained in closed-form, judicious tricks (controlled truncation of infinite summations, interpolations, etc.) are used for the efficient numerical determination of these Fourier transforms. The implemented spectral domain MoM software has been used in the design of a circularly polarized reflectarray antenna based on split rings under the local periodicity condition. The antenna has been analyzed with our spectral domain MoM software, with CST and with HFSS, and good agreement has been found among all sets of results. Our software has proven to be around 27 times faster than CST and HFSS.
\end{abstract}

Index Terms-Multilayered media, moment methods, periodic structures, reflectarrays, circular polarization.

\section{INTRODUCTION}

$\mathbf{R}$ EFLECTARRAY antennas are an interesting alternative to reflector antennas and microstrip arrays owing to their versatile radiation performance, low profile, light weight, ease of fabrication, simplified feeding system, etc. [1]. In the particular case of circular polarization (CP) reflectarrays, the variable rotation technique (VRT) introduced by Huang and Pogorzelski is a design approach that has proven to be very successful [2]. In the frame of this technique, each element of the reflectarray is rotated a different angle to achieve the adequate phase shift in the impinging circularly polarized wave that makes it possible to generate the prescribed radiation pattern after reflection. The VRT requires that the reflection coefficients of the two orthogonal linear components of the impiging wave electric field are of equal magnitude and $180^{\circ}$ out of phase in order to keep the sense of circular polarization (LHCP to LHCP or RHCP to RHCP) after reflection [2], [3]. One reflectarray element that has turned out to be especially suitable for CP applications is that based on split rings. Han et al. used the VRT and single split rings to design a Kaband RHCP reflectarray at $31.75 \mathrm{GHz}$ with measured crosspolarization level below $40 \mathrm{~dB}$ at broadside [4]. Stacked split rings of different size were used in [5] to design a dual frequency RHCP reflectarray operating in the $\mathrm{C}$ and $\mathrm{Ka}$ bands. Smith et al. [6] designed a single layer dual frequency CP reflectarray by using an element with two concentric split rings. The inner rings and the VRT were used to adjust the elements phase at $29.75 \mathrm{GHz}$ for RHCP radiation, while the outer rings and the VRT were used at $19.95 \mathrm{GHz}$ for LHCP radiation. An extension of this work was presented in [7] where the dual frequency CP reflectarray was combined with an FSS made of non-split rings to combine the operation of the reflectarray with that of a second $\mathrm{CP}$ microstrip array antenna working at $\mathrm{L}$ band. Zhao et al. used the single layer element based on two concentric rings and the VRT to design a broadband CP reflectarray at a center frequency of $10 \mathrm{GHz}$ with a bandwidth larger than $30 \%$ for $1-\mathrm{dB}$ gain variation, and a bandwidth larger than $40 \%$ for an axial ratio smaller than 3-dB [8]. In all previous papers dealing with CP applications, the elements of the reflectarrays consist of symmetric split rings with two gaps and one axis of mirror symmetry. Zhang et al. proposed in [9] the use of non-symmetric single gap split rings which can be used as phasing elements for both linear polarization (LP) and CP reflectarray operation. By using this element, a LP reflectarray was designed at $20 \mathrm{GHz}$ with a bandwidth of $23 \%$ for a $1-\mathrm{dB}$ gain variation and an aperture efficiency of $60 \%$.

In the design of a $\mathrm{CP}$ reflectarray antenna made of split rings, the use of the VRT requires to adjust the size of the gaps and their orientation for every single element in order to achieve the required phase shift in the impinging circularly polarized wave, and in order to ensure a phase difference of $180^{\circ}$ in the reflection coefficients of its two orthogonal linear components. When estimating the gap size and their orientation in each element, it is customary to assume that the elements are located in a periodic environment, which is called the local periodicity assumption [10], [11]. The validity of this assumption is justified by the fact that leads to reflectarray designs in which the simulated performance agrees very well with the measured performance [5], [7], [8]. The design of a midsize CP reflectarray antenna made of split rings under the local periodicity assumption requires the analysis of a large number of different multilayered periodic structures owing to the wide variety of gap sizes and orientations that have to be 
adjusted in the split rings of the different reflectarray elements for phase adjustment [7]. Owing to this, a powerful numerical tool is needed for the analysis of these multilayered periodic structures.

In this paper we apply the Method of Moments (MoM) in the spectral domain to the analysis of the scattering of a plane wave by a multilayered periodic structure including concentric split rings in the unit cell. The implemented MoM software is subsequently used in the design of a circularly polarized reflectarray antenna made of split rings. When applying the spectral domain MoM, the 2-D Fourier transforms of the basis functions are expressed as infinite summations of Hankel transforms. This Hankel transform approach was used in the past in conjunction with the spectral domain MoM for the determination of the resonant frequencies of microstrip ring resonators [12], the input impedance and radiation patterns of single and stacked annular-ring microstrip antennas [13], [14], the resonant frequencies of shielded single and coupled microstrip ring resonators [15], [16], and the radar crosssection of concentric annular microstrip rings [17]. However, to the authors' knowledge, it has never been used for microstrip split rings and for multilayered structures in periodic environments. We have only found one paper dealing with the analysis of microstrip split ring resonators in which a magnetic wall approximate model is used for the determination of the resonant frequencies, and where effective dimensions are introduced to account for edge effects [18]. Whereas most papers dealing with the analysis of microstrip ring structures use either magnetic wall cavity mode functions [13], [19] or subsectional piecewise functions [15], [17] as basis functions for the electric current density on the rings, in this paper we have used basis functions that account for edge singularities [16], [19] since these basis functions provide a fast convergence of MoM with respect to the number of basis functions, which implies that only small MoM matrices have to be inverted. Since the Hankel transforms of the edge singularity basis functions cannot be obtained in closed form, we have used especially tailored quadrature rules for their numerical computation, which provide very accurate results with a small number of quadrature points. Also, we have introduced judicious stop criteria for the summation of the infinite series leading to the 2-D Fourier transforms of the basis functions, and we have finally carried out Chebyshev interpolations of these Fourier transforms as a function of the spectral radial variable. As a result of all these strategies, we have developed a very efficient spectral domain MoM approach for the analysis of multilayered periodic structures containing split rings. The spectral domain MoM software has been used in the design of a circularly polarized reflectarray antenna and the results obtained have been compared with results provided by commercial softwares CST $^{\circledR}$ and HFSS ${ }^{\circledR}$. Good agreement has been found between the three sets of results, our software being between one and two orders of magnitude faster than $\operatorname{CST}^{\circledR}$ and $\mathrm{HFSS}^{\circledR}$.

\section{Numerical PROCEDURE}

Figs. 1(a) and (b) show a multilayered periodic structure backed by a ground plane. Concentric conducting split rings are located in each unit cell, and are printed on the upper layer. The center of the rings has been chosen to be coincident with the geometrical center of the unit cell in the $z=0$ plane. The conducting rings and the ground plane will be assumed to be perfect electric conductors (PEC). In a generic case, there would be $R$ concentric rings in the unit cell $(R=2$ in Fig. 1(b)). Also, each ring would be split into $S$ arcs $(S=2$ in Fig. 1(b)) so that the total number of arcs in the unit cell would be $L=R S$ ( $L=4$ in Fig. 1(b)). Let $\rho_{1 l}$ and $\rho_{2 l}$ be the inner and outer radius of the $l$-th arc respectively $(l=1, \ldots, L)$, and let $w_{l}=\rho_{2 l}-\rho_{1 l}$ be the width of the $l$-th arc. The two ends of the $l$-th arc are characterized by the angles $\varphi_{1 l}$ and $\varphi_{2 l}$, such that $\varphi_{1 l}<\varphi_{2 l}$. The angle $\varphi_{k l}(k=1,2 ; l=1, \ldots, L)$ is the angle subtended between the $k-t h$ end of the $l$-th arc and the semiinfinite line directed along the positive $x$ axis with origin at the center of the ring. The angle $\varphi_{k l}$ is taken as positive if, starting from the $x$-directed semi-infinite line, it is drawn in the counterclockwise sense, and is taken as negative if it is drawn in the clockwise sense (e.g., in Fig. 1(b), $\varphi_{21}, \varphi_{12}, \varphi_{22}$, $\varphi_{13}$ and $\varphi_{23}$ are positive, but $\varphi_{11}, \varphi_{14}$ and $\varphi_{24}$ are negative).

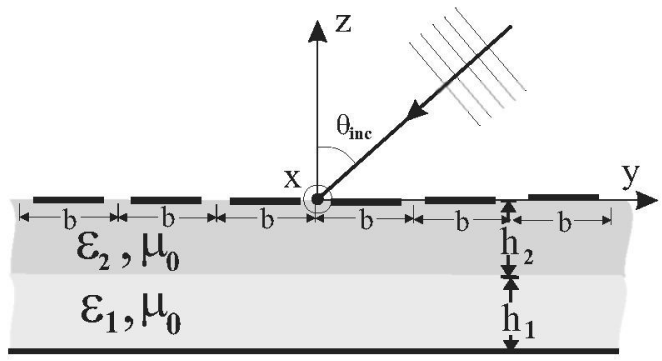

(a)

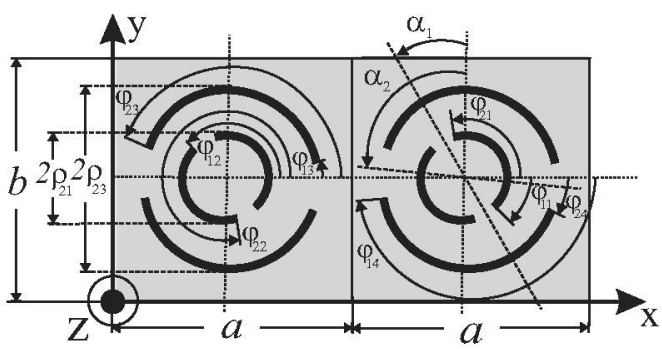

(b)

Fig. 1. Two-layered periodic structure. The unit cell contains two concentric split rings with different axes of mirror symmetry. A plane wave impinges on the multilayered periodic structure. (a) Side view. (b) Top view.

In the particular cases treated in this paper, we will assume that each individual split ring of the periodic structure has an axis of mirror symmetry going through the center of the ring (in the case of Fig. 1(b), this means that $\varphi_{21}-\varphi_{11}=\varphi_{22}-\varphi_{12}$ and $\varphi_{12}-\varphi_{21}=360^{\circ}+\varphi_{11}-\varphi_{22}$, and that $\varphi_{23}-\varphi_{13}=\varphi_{24}$ $-\varphi_{14}$ and $\left.360^{\circ}+\varphi_{14}-\varphi_{23}=\varphi_{13}-\varphi_{24}\right)$. For the $r$-th ring $(r=1, \ldots, R)$, this mirror symmetry axis makes an angle $\alpha_{r}\left(-180^{\circ} \leq \alpha_{r}<180^{\circ}\right)$ with the semi-infinite line directed along the positive $y$ axis with origin at the center of the ring (see Fig. 1(b)). The angle $\alpha_{r}$ is taken as positive if, starting from the $y$-directed semi-infinite line, it is drawn in the counterclockwise sense, and is taken as negative if it is drawn in the clockwise sense (e.g., in Fig. 1(b), $\alpha_{1}$ and $\alpha_{2}$ are both positive). Please note that the restriction introduced by these 
mirror symmetry axes is not necessary in the mathematical derivations presented in the rest of this section.

The concentric split rings of the periodic structure are printed on a multilayered substrate containing $N_{1}$ layers $\left(N_{1}=\right.$ 2 in Fig. 1(a)). The $i$-th layer has a thickness $h_{i}$ and a complex permittivity $\varepsilon_{i}=\varepsilon_{0} \varepsilon_{r, i}\left(1-\mathrm{j} \tan \delta_{i}\right)\left(i=1, \ldots, N_{\mathrm{l}}\right)$. In the following, a time dependence of the type $\mathrm{e}^{\mathrm{j} \omega t}$ will be assumed and suppressed throughout.

As commented in the introduction, the unit cell of the multilayered periodic structure of Figs. 1(a) and (b) has been used as constituent element of several CP reflectarray antennas [6]-[8]. In the design of a CP reflectarray antenna under the local periodicity assumption, each cell is characterized by means of a CP $2 \times 2$ complex reflection matrix, $\overline{\mathbf{R}}_{\mathrm{CP}}$, which relates the RHCP and LHCP components of the reflected and incident electric fields when a plane wave impinges on the cell surrounded by a periodic environment. In this paper, this impinging plane wave is assumed to propagate in an arbitrary incidence direction given by the angular spherical coordinates $\theta_{\text {inc }}$ and $\varphi_{\text {inc }}$ (see Fig. 1(a)). If $E_{\mathrm{RHCP}}^{\mathrm{ref}}$ and $E_{\mathrm{LHCP}}^{\mathrm{ref}}$ are the RHCP and LHCP complex components of the reflected electric field, and $E_{\mathrm{RHCP}}^{\text {inc }}$ and $E_{\mathrm{LHCP}}^{\text {inc }}$ are the RHCP and LHCP complex components of the incident electric field, then

$$
\left(\begin{array}{c}
E_{\mathrm{RHCP}}^{\mathrm{ref}} \\
E_{\mathrm{LHCP}}^{\mathrm{ref}}
\end{array}\right)=\overline{\mathbf{R}}_{\mathrm{CP}} \cdot\left(\begin{array}{c}
E_{\mathrm{RHCP}}^{\mathrm{inc}} \\
E_{\mathrm{LHCP}}^{\text {inc }}
\end{array}\right)
$$

where

$$
\overline{\mathbf{R}}_{\mathrm{CP}}=\left(\begin{array}{ll}
R_{\mathrm{RHCP}, \mathrm{RHCP}} & R_{\mathrm{RHCP}, \mathrm{LHCP}} \\
R_{\mathrm{LHCP}, \mathrm{RHCP}} & R_{\mathrm{LHCP}, \mathrm{LHCP}}
\end{array}\right) .
$$

When writing (1), we assume that the plane wave impinging on the periodic structure does not generate grating lobes after reflection, which requires that the condition $\max (a, b)<$ $\lambda_{0} /\left(1+\sin \theta_{\text {inc }}\right)\left(\lambda_{0}=2 \pi / \omega \sqrt{\mu_{0} \varepsilon_{0}}\right)$ is fulfilled, $a$ and $b$ being the periods of the unit cell in the $x$ and $y$ directions respectively (see Fig. 1(b)).

\section{A. Spectral domain MoM formulation}

In order to obtain the matrix $\overline{\mathbf{R}}_{\mathrm{CP}}$ of (2) for the multilayered periodic structure of Figs. 1(a) and (b), we need to obtain the scattered electric field for impinging plane waves with both RHCP and LHCP polarizations. These scattered electric fields can be obtained in terms of the current density excited at the metallized interface $z=0, \mathbf{J}(x, y)$, by the impinging waves. Let $\mathbf{E}^{\mathrm{ms}}(x, y, z)$ be the electric field generated in all space by a plane wave impinging on the multilayered substrate of Figs. 1(a) and (b) in the absence of the conducting split rings. The current density $\mathbf{J}(x, y)$ induced on the split rings will be the solution of the following electric field integral equation (EFIE) [20]

$$
\begin{aligned}
& \hat{\mathbf{z}} \times\left[\mathbf{E}^{\mathrm{ms}}(x, y, z=0)+\sum_{m=-\infty}^{+\infty} \sum_{n=-\infty}^{+\infty} \int_{S_{m n}}\right. \\
& \left.\overline{\mathbf{G}}^{E}\left(x-x^{\prime}, y-y^{\prime}, z=0, z^{\prime}=0\right) \cdot \mathbf{J}\left(x^{\prime}, y^{\prime}\right) d x^{\prime} d y^{\prime}\right]=\mathbf{0} \\
& (x, y) \in S_{00}
\end{aligned}
$$

where $S_{m n}(m, n=\ldots,-1,0,1, \ldots)$ is the metallized portion of the $z=0$ plane within the $m n$-th periodic unit cell, and $\overline{\mathbf{G}}^{E}$ is the non-periodic dyadic Green's function of the multilayered substrate [21]. Since $\mathbf{J}(x, y)$ is a Floquet-periodic function of $x$ and $y$, in order to solve the EFIE of (3), we only need to determine $\mathbf{J}(x, y)$ within one unit cell, e. $\mathrm{g}$., the cell $C_{00}$ covering the rectangular domain $\{0 \leq x \leq a ; 0 \leq y \leq b\}$. For that purpose, we expand $\mathbf{J}(x, y)$ in $C_{00}$ in terms of known basis functions $\mathbf{J}_{l q}(x, y)\left(l=1, \ldots, L ; q=1, \ldots, N_{\mathrm{b}}\right)$ as shown below

$$
\mathbf{J}(x, y)=\sum_{l=1}^{L} \sum_{q=1}^{N_{\mathrm{b}}} c_{l q} \mathbf{J}_{l q}(x, y)
$$

where $\mathbf{J}_{l q}(x, y)\left(q=1, \ldots, N_{\mathrm{b}}\right)$ is the set of $N_{\mathrm{b}}$ basis functions used to approximate the current density on the $l$-th arc of the $C_{00}$ unit cell. When (4) is introduced in (3) and Galerkin's version of MoM is applied, the following system of linear equations is obtained for the unknown coefficients $c_{l_{q}}$ [20]

$$
\sum_{l=1}^{L} \sum_{q=1}^{N_{\mathrm{b}}} \Gamma_{k l, p q} c_{l q}=e_{k p} \quad\left(k=1, \ldots, L ; p=1, \ldots, N_{\mathrm{b}}\right) .
$$

If we invoke Parseval's identity for 2-D Fourier transforms, the MoM matrix entries $\Gamma_{k l, p q}$ of (5) can be expressed in the spectral domain as double infinite summations given by [22]

$$
\begin{aligned}
\Gamma_{k l, p q}= & a b \sum_{m=-\infty}^{+\infty} \sum_{n=-\infty}^{+\infty}\left[\left(\widetilde{J}_{k p}^{\mathrm{d}}\left(k_{x m}, k_{y n}\right)\right)^{*}\right]^{t} \\
& \cdot \overline{\mathbf{G}}^{E, \mathrm{c}}\left(k_{x}=k_{x m}, k_{y}=k_{y n}, z=0, z^{\prime}=0\right) \\
& \cdot \widetilde{\mathbf{J}}_{l q}^{\mathrm{d}}\left(k_{x m}, k_{y n}\right)
\end{aligned}
$$

where $\overline{\overline{\mathbf{G}}}^{E, \mathrm{c}}\left(k_{x}, k_{y}, z=0, z^{\prime}=0\right)$ is the continuous 2-D Fourier transform of $\overline{\mathbf{G}}^{E}\left(x, y, z=0, z^{\prime}=0\right)$ [21], $k_{x m}=$ $k_{0} \sin \theta_{\mathrm{inc}} \cos \varphi_{\mathrm{inc}}+2 \pi m / a, k_{y n}=k_{0} \sin \theta_{\mathrm{inc}} \sin \varphi_{\mathrm{inc}}+2 \pi n / b$, $k_{0}=2 \pi / \lambda_{0}$ and $\widetilde{\mathbf{J}}_{l q}^{\mathrm{d}}\left(k_{x m}, k_{y n}\right)$ is the discrete 2-D Fourier transform of $\mathbf{J}_{l q}(x, y)$, which is given by

$$
\widetilde{\mathbf{J}}_{l q}^{\mathrm{d}}\left(k_{x m}, k_{y n}\right)=\frac{1}{a b} \int_{S_{00}} \mathbf{J}_{l q}(x, y) \mathrm{e}^{-\mathrm{j}\left(k_{x m} x+k_{y n} y\right)} d x d y
$$

Finally, the coefficients $e_{k p}$ of the system of equations (5) can be obtained in the spectral domain as

$$
\begin{aligned}
e_{k p}= & -a b\left[\left(\widetilde{\mathbf{J}}_{k p}^{\mathrm{d}}\left(k_{x 0}, k_{y 0}\right)\right)^{*}\right]^{t} \cdot \mathbf{E}^{\mathrm{ms}}(x, y, z=0) \\
& \times \mathrm{e}^{-\mathrm{j} k_{0}\left(\sin \theta_{\mathrm{inc}} \cos \varphi_{\mathrm{inc}} x+\sin \theta_{\mathrm{inc}} \sin \varphi_{\mathrm{inc}} y\right)}
\end{aligned}
$$

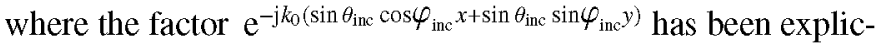
itly included in (8) to absorb the dependence of $\mathbf{E}^{\mathrm{ms}}(x, y, z=0)$ on $x$ and $y$.

Equations (5) to (8) provide the spectral domain MoM formulation for the determination of the vector function $\mathbf{J}(x, y)$ of (3) and (4). The problem with this formulation is that the discrete 2-D Fourier transforms $\widetilde{\mathbf{J}}_{l q}^{\mathrm{d}}\left(k_{x m}, k_{y n}\right)$ of (6) (defined in (7)) cannot be obtained in closed form, even for the simplest choice of basis functions in (4) (i.e., a constant value for the two components of $\mathbf{J}_{l q}(x, y)$ on the surface of the split rings of Figs. 1(a) and (b)). In the following subsection, we will see how to tackle this problem. 


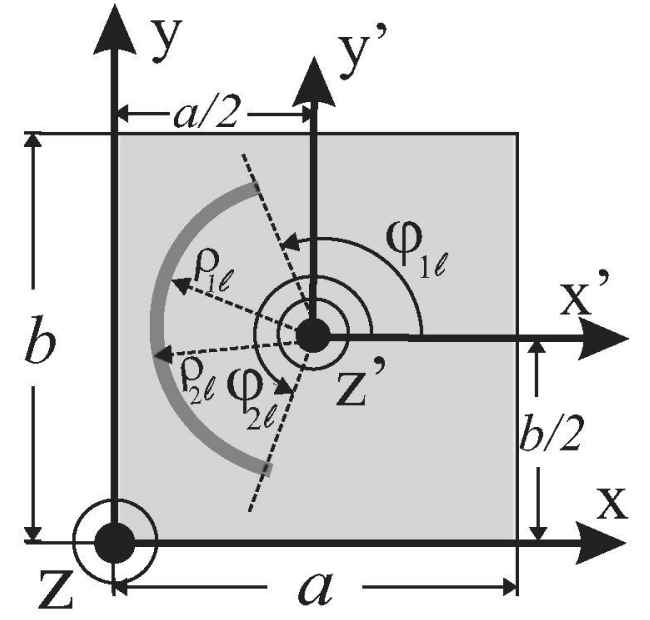

Fig. 2. Top view of the $l$-th arc in the left unit cell of Fig. 1(b). Radial and angular dimensions of the $l$-th arc are shown.

\section{B. Basis functions and 2-D Fourier transforms}

Fig. 2 shows the $l$-th arc $(l=1, \ldots, L=4)$ of the unit cell $C_{00}$ of Fig. 1(b). In Fig. 2 we have defined a shifted system of coordinates $\left\{x^{\prime}, y^{\prime}, z^{\prime}\right\}$ with origin at the center of the unit cell in the plane $z=0$. We are going to introduce shifted polar coordinates $\rho^{\prime}$ and $\varphi^{\prime}$, which are related with the original coordinates $x$ and $y$ as

$$
\begin{aligned}
& x=\frac{a}{2}+\rho^{\prime} \cos \varphi^{\prime} \\
& y=\frac{b}{2}+\rho^{\prime} \sin \varphi^{\prime}
\end{aligned}
$$

The unit vectors linked to the shifted polar coordinates are given by

$$
\begin{aligned}
& \hat{\rho}^{\prime}=\cos \varphi^{\prime} \hat{\mathbf{x}}+\sin \varphi^{\prime} \hat{\mathbf{y}} \\
& \hat{\varphi}^{\prime}=-\sin \varphi^{\prime} \hat{\mathbf{x}}+\cos \varphi^{\prime} \hat{\mathbf{y}}
\end{aligned}
$$

In the split rings used in $\mathrm{CP}$ reflectarray antennas, the condition $w_{l}=\rho_{2 l}-\rho_{1 l} \ll\left(\rho_{1 l}+\rho_{2 l}\right) / 2$ (see Fig. 2 ) is usually fulfilled (i.e., the width of the rings is usually much smaller than their mean radius) [6]-[8]. Assuming this condition holds, we are going to neglect the radial component of the basis functions $\mathbf{J}_{l q}(x, y)$ of $(4), \mathbf{J}_{l q}(x, y) \cdot \hat{\boldsymbol{\rho}}^{\prime}$, by comparison with their azimuthal component, $\mathbf{J}_{l q}(x, y) \cdot \hat{\varphi}^{\prime}$. Also, we will assume that the azimuthal component of $\mathbf{J}_{l q}(x, y)$ can be factored in terms of independent functions of $\rho^{\prime}$ and $\varphi^{\prime}$, i.e., we will assume that the functions $\mathbf{J}_{t q}(x, y)$ can all be mathematically written as

$$
\begin{aligned}
\mathbf{J}_{l q}(x, y)= & f_{l}\left(\rho^{\prime}\right) g_{l q}\left(\varphi^{\prime}\right) \hat{\varphi}^{\prime} \\
& \left(\rho_{1 l}<\rho^{\prime}<\rho_{2 l} ; \varphi_{1 l}<\varphi^{\prime}<\varphi_{2 l}\right)
\end{aligned}
$$

As we will see in Section III, the assumption shown in (13) is justified by the fact that leads to values of the matrix $\overline{\mathbf{R}}_{\mathrm{CP}}$ in (2) that match the values provided by the commercial software $\mathrm{CST}^{\circledR}$. Although the radial component, $\mathbf{J}_{l_{q}}(x, y) \cdot \hat{\boldsymbol{\rho}}^{\prime}$, of $\mathbf{J}_{l_{q}}(x, y)$ has been neglected in (13), we would like to point out that this component can be easily accommodated in the mathematical formulation presented in the rest of this subsection.
Since the functions $g_{l q}\left(\varphi^{\prime}\right) \hat{\varphi}^{\prime}$ of (13) are periodic functions of $\varphi^{\prime}$ with period $2 \pi$, these functions can be expanded as Fourier series of $\varphi^{\prime}$ as shown below

$$
g_{l q}\left(\varphi^{\prime}\right) \hat{\boldsymbol{\varphi}}^{\prime}=\sum_{i=-\infty}^{+\infty} \widetilde{\mathbf{g}}_{l q}^{i} \mathrm{e}^{\mathrm{j} i \varphi^{\prime}} \quad\left(\varphi_{1 l}<\varphi^{\prime}<\varphi_{2 l}\right)
$$

where

$$
\overrightarrow{\mathbf{g}}_{l q}^{i}=\frac{1}{2 \pi} \int_{\varphi_{1 l}}^{\varphi_{2 l}} g_{l_{q}}\left(\varphi^{\prime}\right) \hat{\varphi}^{\prime} \mathrm{e}^{-\mathrm{j} i \varphi^{\prime}} d \varphi^{\prime}
$$

Now, let us introduce the polar discrete spectral coordinates $k_{\rho, m n}$ and $k_{\varphi, m n}$ given by

$$
\begin{aligned}
& k_{\rho, m n}=\sqrt{\left(k_{x m}\right)^{2}+\left(k_{y n}\right)^{2}} \\
& k_{\varphi, m n}=\arctan \left(\frac{k_{y n}}{k_{x m}}\right)
\end{aligned}
$$

If (9) to (14), (16) and (17) are introduced in (7), after some manipulations, it is possible to express $\widetilde{\mathbf{J}}_{l q}^{\mathrm{d}}\left(k_{x m}, k_{y n}\right)$ as

$$
\begin{aligned}
& \widetilde{\mathbf{J}}_{l q}^{\mathrm{d}}\left(k_{x m}, k_{y n}\right)=\frac{2 \pi \mathrm{e}^{-\mathrm{j}\left(\frac{k_{m m a+k m b}}{2}\right)}}{a b} \\
& \times\left\{\widetilde{\mathbf{g}}_{q q}^{0} \tilde{f}_{l}^{0}\left(k_{\rho}=k_{\rho, m n}\right)+\sum_{i=1}^{+\infty} \mathrm{e}^{-\mathrm{j} i \pi / 2} \tilde{f}_{l}^{i}\left(k_{\rho}=k_{\rho, m n}\right)\right. \\
& \left.\left[\mathrm{e}^{\mathrm{i} k k_{\varphi, m}} \widetilde{\mathbf{g}}_{l q}^{i}+\mathrm{e}^{-\mathrm{j} i k \varphi, m n}\left(\widetilde{\mathbf{g}}_{l q}^{i}\right)^{*}\right]\right\}
\end{aligned}
$$

where $\tilde{f}_{l}^{i}\left(k_{\rho}\right)(i=0,1, \ldots)$ are Hankel transforms of order $i$ of the functions $f_{l}\left(\rho^{\prime}\right)$ of (13), which can be expressed as

$$
\tilde{f}_{l}^{i}\left(k_{\rho}\right)=\int_{\rho_{1 l}}^{\rho_{2 l}} J_{i}\left(k_{\rho} \rho^{\prime}\right) \rho^{\prime} f_{l}\left(\rho^{\prime}\right) d \rho^{\prime},
$$

and where the function $J_{i}(\cdot)$ of (19) is a Bessel functions of first kind and order $i$.

Although the expression (18) for the computation of $\widetilde{\mathbf{J}}_{l q}^{\mathrm{d}}\left(k_{x m}, k_{y n}\right)$ looks simpler than (7), it presents two drawbacks. First, the functions $\tilde{f}_{l}^{i}\left(k_{\rho}\right)$ cannot be obtained in closed form, even for the simplest choice of $f_{l}\left(\rho^{\prime}\right)$ (e.g., a constant value in the interval $\rho_{1 l}<\rho^{\prime}<\rho_{2 l}$ ). Second, (18) involves the determination of an infinite series, which has a deleterious impact on the computation of $\widetilde{\mathbf{J}}_{l q}^{\mathrm{d}}\left(k_{x m}, k_{y n}\right)$ by means of (18).

In this paper, the functions chosen for $\mathbf{J}_{l q}(x, y)$ are entire domain basis functions which account for the singularities of $\mathbf{J}(x, y)$ at the edges of the arcs of Fig. 1(b). It is well known that these functions ensure a fast convergence of the spectral domain MoM with respect to the number of basis functions $\left(L N_{\mathrm{b}}\right.$ in (4)) [16], [19], [20], as will be demonstrated in Section 
III. The particular functions chosen for $f_{l}\left(\rho^{\prime}\right)$ and $g_{l q}\left(\varphi^{\prime}\right)$ can be written as

$$
\begin{aligned}
& f_{l}\left(\rho^{\prime}\right)= \frac{1}{\sqrt{1-\left(\frac{2}{\rho_{2 l}-\rho_{1 l}}\left[\rho^{\prime}-\frac{\rho_{2 l}+\rho_{1 l}}{2}\right]\right)^{2}}} \\
& g_{l q}\left(\varphi^{\prime}\right)= \sqrt{1-\left(\frac{2}{\varphi_{2 l}-\varphi_{1 l}}\left[\varphi^{\prime}-\frac{\varphi_{2 l}+\varphi_{1 l}}{2}\right]\right)^{2}} \\
& \times U_{q-1}\left(\frac{2}{\varphi_{2 l}-\varphi_{1 l}}\left[\varphi^{\prime}-\frac{\varphi_{2 l}+\varphi_{1 l}}{2}\right]\right) \\
&\left(l=1, \ldots, L ; q=1, \ldots, N_{\mathrm{b}}\right)
\end{aligned}
$$

where $U_{q-1}(\cdot)$ is a Chebyshev polynomial of second kind and degree $q-1$.

The integrals of (15) can be obtained in closed form for the functions $g_{l q}\left(\varphi^{\prime}\right)$ of $(21)$, and their expression is given in the Appendix A. However, the integrals of (19) cannot be obtained in closed form for the functions $f_{l}\left(\rho^{\prime}\right)$ of $(20)$. Fortunately, we have checked these latter integrals can be numerically obtained within very reasonable CPU times by means of Gauss-Chebyshev quadrature rules. In particular, when the functions $f_{l}\left(\rho^{\prime}\right)$ of (20) are introduced in (19), the integrals can be rewritten as

$$
\tilde{f}_{l}^{i}\left(k_{\rho}\right)=\int_{-1}^{+1} \frac{h_{l}^{i}\left(k_{\rho}, t\right)}{\sqrt{1-t^{2}}} d t
$$

where

$$
\begin{aligned}
h_{l}^{i}\left(k_{\rho}, t\right)= & {\left[\frac{\rho_{2 l}-\rho_{1 l}}{2}\right] J_{i}\left(k_{\rho}\left\{\left[\frac{\rho_{2 l}-\rho_{1 l}}{2}\right] t+\left[\frac{\rho_{2 l}+\rho_{1 l}}{2}\right]\right\}\right) } \\
& \times\left\{\left[\frac{\rho_{2 l}-\rho_{1 l}}{2}\right] t+\left[\frac{\rho_{2 l}+\rho_{1 l}}{2}\right]\right\}
\end{aligned}
$$

The integrals of (22) are amenable to be computed by means of Gauss-Chebyshev quadrature rules [23, Eqn. 25.4.38]. In particular, the integrals should be approximately obtained by means of the closed-form formula

$$
\tilde{f}_{l}^{i}\left(k_{\rho}\right) \approx \sum_{j=1}^{N_{\mathrm{qp}}} \eta_{j} h_{l}^{i}\left(k_{\rho}, t=t_{j}\right)
$$

where $\eta_{j}$ and $t_{j}$ are given by $\eta_{j}=\pi / N_{\mathrm{qp}}$ and $t_{j}=$ $\cos \left((2 j-1) \pi / 2 N_{\mathrm{qp}}\right)\left(j=1, \ldots, N_{\mathrm{qp}}\right)$. Since the functions $h_{l}^{i}\left(k_{\rho}, t\right)$ are non-singular smooth functions of $t$ in the interval $-1 \leq t \leq+1$, a low number of quadrature points, $N_{\mathrm{qp}}$, should suffice to obtain $\tilde{f}_{l}^{i}\left(k_{\rho}\right)$ with a large accuracy. This has been confirmed by numerical simulations, as will be shown in Section III.

Concerning the infinite series of (18), its convergence is strongly dependent on the value of $k_{\rho, m n}$. The larger the value of $k_{\rho, m n}$, the larger the number of terms that has to be retained in the series of (18) for an accurate determination of $\widetilde{\mathbf{J}}_{l q}^{\mathrm{d}}\left(k_{x m}, k_{y n}\right)$. Let us see how the convergence of this infinite series depends on $k_{\rho, m n}$. If we assume that $a \approx b$ (which holds in practical cases for most reflectarray antennas), by virtue of the mean value theorem for definite integrals, we can write that (see [23, Eqn. 9.3.1])

$$
\begin{aligned}
& \left|\tilde{f}_{l}^{i}\left(k_{\rho}=k_{\rho, m n}\right)\right|_{|i| \gg} \propto\left|J_{i}\left(k_{\rho, m n}\left(\frac{\rho_{2 l}+\rho_{1 l}}{2}\right)\right)\right|_{|i| \gg} \\
& \approx\left|J_{i}\left(\frac{k_{\rho, m n} a}{2}\right)\right|_{|i|>\gg} \propto\left(\frac{\mathrm{e} k_{\rho, m n} a}{4 i}\right)^{i}=\mathrm{e}^{i \ln (\mathrm{e} k \rho, m n a / 4 i)}
\end{aligned}
$$

which indicates that the series of (18) has an exponential convergence provided $\mathrm{e} k_{\rho, m n} a / 4 i \leq 1$, i. e., provided $i \geq \mathrm{e} k_{\rho, m n} a / 4$. Numerical simulations have shown that the infinite series of (18) has to be added in the interval $1 \leq$ $i \leq \mathrm{e} k_{\rho, m n} a / 4+8$ for an accurate estimation of $\widetilde{\mathbf{J}}_{l q}^{\mathrm{d}}\left(k_{x m}, k_{y n}\right)$. Therefore, the larger $k_{\rho, m n}$, the larger the number of terms to be retained in the series.

In practice, the computation of $\Gamma_{k l, p q}$ requires to truncate the infinite summations of (6) within the intervals $-N_{\max } \leqslant$ $m, n \leqslant+N_{\max }$. If we assume that $a \approx b$, the maximum value of $k_{\rho, m n}$ we will have to use in (6) after the truncation will be (see (16))

$$
k_{\rho, m n}^{\max }=\sqrt{\left(k_{x m}^{\max }\right)^{2}+\left(k_{y n}^{\max }\right)^{2}} \approx \sqrt{2} \frac{2 \pi N_{\max }}{a}
$$

which means the maximum number of terms that will be necessary to retain in the infinite series of (18) will be

$$
i_{\text {max }}=\frac{\mathrm{e} k_{\rho, m n}^{\max } a}{4}+8 \approx \frac{\pi \mathrm{e} N_{\max }}{\sqrt{2}}+8
$$

For the cases treated in the results section, we have used $N_{\max }=50$, which leads to $i_{\max } \approx 310$ when using (27). For our purposes, this is the worst case scenario in the evaluation of (18), i.e., any evaluation of $\widetilde{\mathbf{J}}_{l q}^{\mathrm{d}}\left(k_{x m}, k_{y n}\right)$ within $-N_{\max } \leqslant$ $m, n \leqslant+N_{\max }$ will require to retain at most 310 terms in the computation of the infinite series of (18) when $N_{\max }=50$.

Apart from the analytical stop criterium established above for the summation of the series of (18) $\left(1 \leq i \leq \mathrm{e} k_{\rho, m n} a / 4+8\right)$, one additional fact that helps to reduce the CPU time required in the computation of this series is a well known property of Bessel functions, according to which $J_{i}(x) \approx 0$ if $x<x_{\text {th }}=C_{\text {th }} i$ when $i>N_{\text {th }}$. Numerical simulations show this property holds for $N_{\text {th }}$ around 10 and $C_{\text {th }}$ around 1. According to (19), this property of Bessel functions implies that $\tilde{f}_{l}^{i}\left(k_{\rho}=k_{\rho, m n}\right) \approx 0$ in (18) when $i>N_{\text {th }}$ and $\left(k_{\rho, m n} a\right) / 2<C_{\text {th }} i$, which makes it unnecessary the evaluation of a large number of summation terms in the numerical computation of the series leading to $\widetilde{\mathbf{J}}_{l q}^{\mathrm{d}}\left(k_{x m}, k_{y n}\right)$ by means of (18). In Section III we will present a numerical method that makes it possible to easily estimate two new quantities $d_{\mathrm{th}}(l)$ and $b_{\mathrm{th}}(l)$ (which are a function of $\rho_{1 l}$ and $\left.\rho_{2 l}\right)$ for which $\tilde{f}_{l}^{i}\left(k_{\rho}\right) \approx 0$ if $k_{\rho} a<d_{\text {th }}(l)\left(i-b_{\text {th }}(l)\right)$ and $i \geq b_{\mathrm{th}}(l)$. According to this, it will only be necessary to compute $\tilde{f}_{l}^{i}\left(k_{\rho}=k_{\rho, m n}\right)$ in the interval

$$
k_{\rho, m n}^{\min }(i)=\frac{d_{\mathrm{th}}(l)\left(i-b_{\mathrm{th}}(l)\right)}{a} \leq k_{\rho} \leq k_{\rho, m n}^{\max }
$$

when $i \geq b_{\text {th }}(l)$. For $i<b_{\text {th }}(l)$, we will have to compute $\tilde{f}_{l}^{i}\left(k_{\rho}=\right.$ $\left.k_{\rho, m n}\right)$ in the interval $k_{\rho, m n}^{\min }(i)=0 \leq k_{\rho} \leq k_{\rho, m n}^{\max }$.

The integrals $\tilde{f}_{l}^{i}\left(k_{\rho}\right)$ of (19) are a function of $k_{\rho}, \rho_{1 l}$ and $\rho_{2 l}$ for each value of $i$. In most applications of reflectarray antennas the distances $\rho_{1 l}$ and $\rho_{2 l}(l=1, \ldots, L)$ remain fixed from element to element and the angles $\varphi_{k l}(k=1, \ldots, S ; l=$ 
$1, \ldots, L)$ and $\alpha_{r}(r=1, \ldots, R)$ are varied to obtain the required phase shifts. So, a large amount of CPU time can be saved in the design of a large reflectarray antenna if the functions $\tilde{f}_{l}^{i}\left(k_{\rho}\right)$ $\left(l=1, \ldots, L ; i=0, \ldots, i_{\max }\right)$ are interpolated as a function of $k_{\rho}$ for fixed values of $\rho_{1 l}$ and $\rho_{2 l}$, and the interpolations are subsequently used when adjusting the angles $\varphi_{k l}$ and $\alpha_{r}$ in each element. The interpolations have to be performed in the interval $k_{\rho, m n}^{\min }(i) \leq k_{\rho} \leq k_{\rho, m n}^{\max }$ for each value of $l$ and $i$. In practice, we have divided the required interpolation intervals into $N_{\text {int }}$ subintervals, and we have used Chebyshev polynomials up to seventh-degree in the interpolation in each subinterval. In Section III we will see that a maximum number of $N_{\text {int }}=80$ subintervals suffices to obtain interpolated values of $\tilde{f}_{l}^{i}\left(k_{\rho}\right)$ within four significant figures, and that the number of required subintervals $N_{\text {int }}$ decreases as $i$ increases, which is a consequence of the increase of $k_{\rho, m n}^{\min }(i)$ (and therefore, of the reduction of the width of the original interpolation interval) as $i$ increases.

\section{NuMERICAL RESUlTS AND VALIDATIONS}

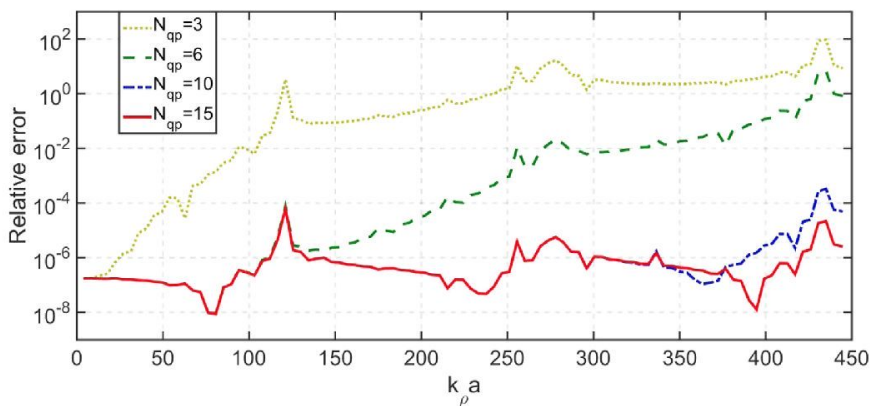

(a)

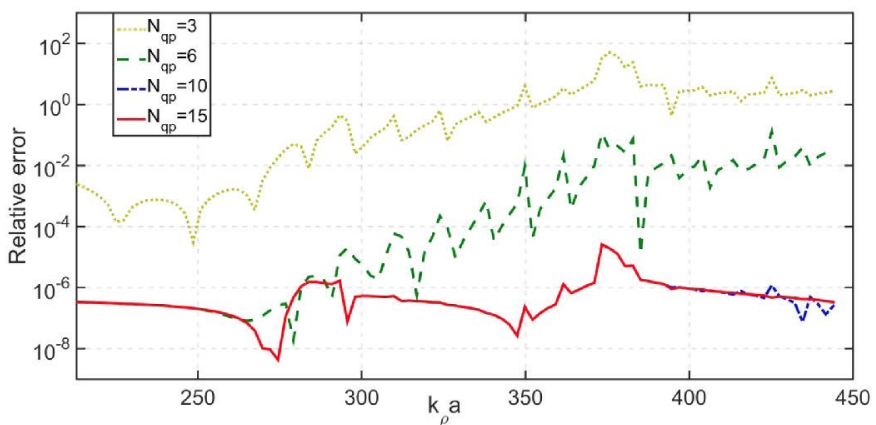

(b)

Fig. 3. Magnitude of the relative errors in the computation of (a) $\tilde{f}_{l}^{0}\left(k_{\rho}\right)$ and (b) $\tilde{f}_{l}^{100}\left(k_{\rho}\right)$ by means of (24). The relative errors are plotted as a function of the number of quadrature points $N_{\mathrm{qp}}$ employed in (24). Parameters: $a=b=5$ $\mathrm{mm} ; \rho_{1 l}=1.85 \mathrm{~mm} ; \rho_{2 l}=2.05 \mathrm{~mm}$.

Figs. 3(a) and (b) show the relative errors made in the evaluation of $\tilde{f}_{l}^{0}\left(k_{\rho}\right)$ and $\tilde{f}_{l}^{100}\left(k_{\rho}\right)$ by means of (24) as a function of $k_{\rho}$, and as a function of the number of Gauss-Chebyshev quadrature points $N_{\mathrm{qp}}$ employed in (24). The reference values used for $\tilde{f}_{l}^{0}\left(k_{\rho}\right)$ and $\tilde{f}_{l}^{100}\left(k_{\rho}\right)$ have been computed by means of the double exponential quadrature rule with 203 integration points (level of the quadrature rule $M=5$ ), since this is a numerical procedure which is particularly accurate when used in the integration of functions with integrable singularities at the end points of the integration interval [24]. Please note that a value of $N_{\mathrm{qp}}$ equal to 10 suffices to provide 4 significant figures in the values of $\tilde{f}_{l}^{0}\left(k_{\rho}\right)$ and $\tilde{f}_{l}^{100}\left(k_{\rho}\right)$ in nearly all cases, which indicates that (24) provides values that are accurate enough for $\tilde{f}_{l}^{i}\left(k_{\rho}\right)$ within a very low CPU time consumption. In the rest of the results of this Section, we will always use $N_{\mathrm{qp}}=10$ in (24).

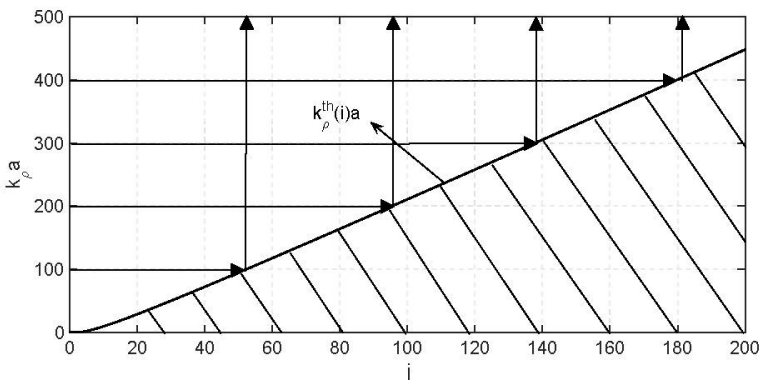

(a)

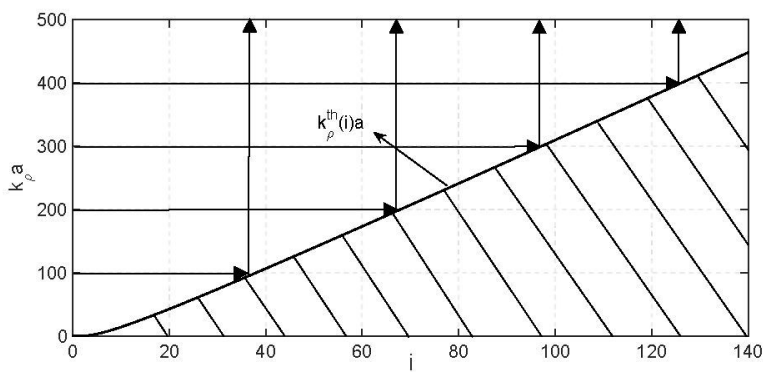

(b)

Fig. 4. The solid line $k_{\rho}^{\text {th }}(i) a$ stands for the threshold values of $k_{\rho} a$ for which $\tilde{f}_{l}^{i}\left(k_{\rho}\right)$ is negligible if $k_{\rho} a<k_{\rho}^{\mathrm{th}}(i) a$, and $\tilde{f}_{l}^{i}\left(k_{\rho}\right)$ is non-negligible if $k_{\rho} a>k_{\rho}^{\mathrm{th}}(i) a, k_{\rho}^{\mathrm{th}}(i) a$ is plotted as a function of $i$. Parameters: $a=b=5 \mathrm{~mm}$; $\rho_{1 l}=1.85 \mathrm{~mm}$ and $\rho_{2 l}=2.05 \mathrm{~mm}$ in (a); $\rho_{1 l}=1.20 \mathrm{~mm}$ and $\rho_{2 l}=1.40 \mathrm{~mm}$ in (b).

In Figs. 4(a) and (b) we plot the threshold values $k_{\rho}^{\text {th }}(i)$ as a function of $i$, where $k_{\rho}^{\text {th }}(i)$ are the values of $k_{\rho}$ for which $\left|\tilde{f}_{l}^{i}\left(k_{\rho}\right) / \tilde{f}_{l}^{0}\left(k_{\rho}\right)\right|<10^{-4}$ if $k_{\rho}<k_{\rho}^{\text {th }}(i)$ and $\left|\tilde{f}_{l}^{i}\left(k_{\rho}\right) / \tilde{f}_{l}^{0}\left(k_{\rho}\right)\right|>10^{-4}$ if $k_{\rho}>k_{\rho}^{\text {th }}(i)$. Therefore, for every value of $i$, the threshold value $k_{\rho}^{\text {th }}(i)$ indicates the value of $k_{\rho}$ below which $\tilde{f}_{l}^{i}\left(k_{\rho}\right)$ can be considered to be vanishing. Please note the plot of $k_{\rho}^{\text {th }}(i)$ as a function of $i$ fits very well to a straight line. So we have carried out a linear least squares fitting of $k_{\rho}^{\text {th }}(i)$ as a function of $i$ given by

$$
k_{\rho}^{\mathrm{th}}(i) a \approx d_{\mathrm{th}}(l)\left(i-b_{\mathrm{th}}(l)\right)
$$

which should be valid for $i \geq b_{\mathrm{th}}(l)$. For the least squares fitting, we have used $N_{\text {sam }}$ samples of $i$ in ascending order, $i_{k}\left(k=1, \ldots, N_{\text {sam }}\right)$ the first sample being $i_{1}=20$. In the case of Fig. 4(a), it turns out that $d_{\mathrm{th}}(l) \approx 2.343$ and $b_{\text {th }}(l) \approx 9.484$ with a coefficient of determination $r^{2}=0.9999$. In the case of Fig. $4(\mathrm{~b})$, it turns out that $d_{\mathrm{th}}(l) \approx 3.388$ and $b_{\mathrm{th}}(l) \approx 8.371$ with a coefficient of determination $r^{2}=0.9998$. These results indicate that the values of $d_{\mathrm{th}}(l)$ and $b_{\mathrm{th}}(l)$ are slightly dependent on the values of the inner and outer radii of the rings, $\rho_{1 l}$ and $\rho_{2 l}$, which are the endpoints of the integration 
interval of (19). Since the fitting of $k_{\rho}^{\text {th }}(i)$ to a straight line for $i \geq b_{\text {th }}(l)$ is very good, in practice a small value of the number of samples of $k_{\rho}^{\text {th }}(i), N_{\mathrm{sam}}$, suffices for the determination of $d_{\mathrm{th}}(l)$ and $b_{\mathrm{th}}(l)$. Once these values of $d_{\mathrm{th}}(l)$ and $b_{\mathrm{th}}(l)$ are known, one can use (28) to estimate the interval of values of $k_{\rho}$ in which the functions $\tilde{f}_{L}^{i}\left(k_{\rho}\right)$ present non-negligible values with a view to computing $\mathbf{J}_{l q}^{\mathrm{d}}\left(k_{x m}, k_{y n}\right)$ via (18).

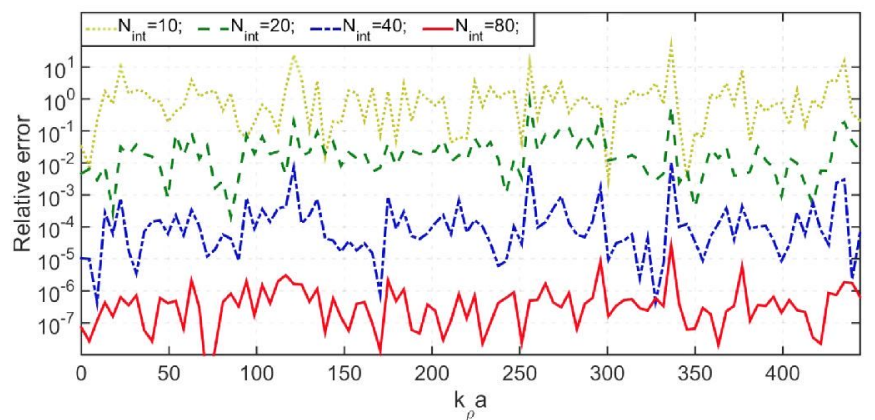

(a)

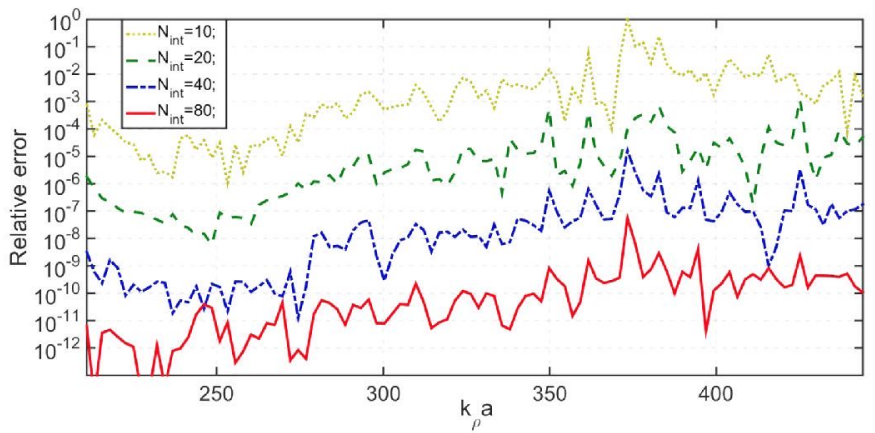

(b)

Fig. 5. Magnitude of the relative errors in the interpolation of (a) $\tilde{f}_{l}^{0}\left(k_{\rho}\right)$ and (b) $\tilde{f}_{l}^{100}\left(k_{\rho}\right)$ as a function of the number of subintervals $N_{\text {int }}$ used in the interpolation. Parameters: $a=b=5 \mathrm{~mm} ; \rho_{1 l}=1.85 \mathrm{~mm} ; \rho_{2 l}=2.05 \mathrm{~mm}$.

Figs. 5(a) and (b) show the errors made in the interpolation of $\tilde{f}_{l}^{\emptyset}\left(k_{\rho}\right)$ and $\tilde{f}_{l}^{100}\left(k_{\rho}\right)$ in the interval $k_{\rho, m n}^{\min }(i) \leq k_{\rho} \leq k_{\rho, m n}^{\max }$ when this interval is divided into $N_{\text {int }}$ subintervals of equal width, and Chebyshev polynomials up to seventh-degree are subsequently used to carry out the interpolation in each subinterval. Please note that whereas 80 subintervals are required for the determination of $\tilde{f}_{l}^{0}\left(k_{\rho}\right)$ with an accuracy of 4 significant figures in the whole interval $k_{\rho, m n}^{\min }(i) \leq k_{\rho} \leq k_{\rho, m n}^{\max }$, only 40 subintervals are required for the determination of $\tilde{f}_{l}^{100}\left(k_{\rho}\right)$ with the same accuracy. The explanation for this is that whereas the whole interpolation interval for $\tilde{f}_{l}^{0}\left(k_{\rho}\right)$ is roughly $0 \leq k_{\rho} a \lesssim 450$, the interpolation interval for $\tilde{f}_{l}^{100}\left(k_{\rho}\right)$ is much smaller, $200 \leqslant k_{\rho} a \leqslant 450$, which is a consequence of the fact that $\tilde{f}_{l}^{100}\left(k_{\rho}\right)$ is negligible for $k_{\rho} a \lesssim 200$ as shown in Fig.4(a).

Fig. 6(b) shows the phase curves required for the design of a CP reflectarray antenna (see (2)) made of single split rings (see Fig. 6(a)) at a frequency of $19.95 \mathrm{GHz}$. By virtue of the VRT of [2], a linear phase variation is obtained for both $\angle R_{\mathrm{RHCP}, \mathrm{RHCP}}$ and $\angle R_{\mathrm{LHCP}, \mathrm{LHCP}}$ as a function of $\alpha_{2}$, which is the rotation angle of the mirror symmetry axis of the split ring. In order to keep the sense of $\mathrm{CP}$ after reflection in a reflectarray antenna, we need a phase difference of $180^{\circ}$

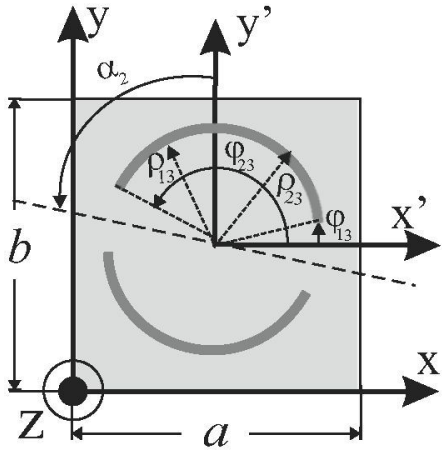

(a)

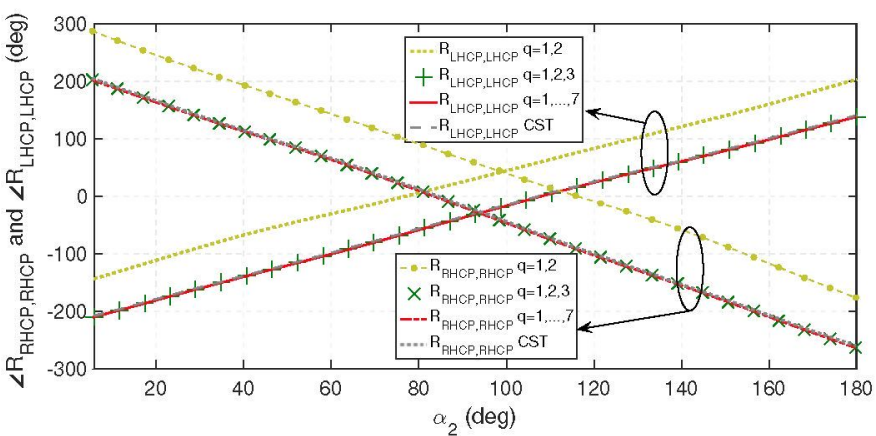

(b)

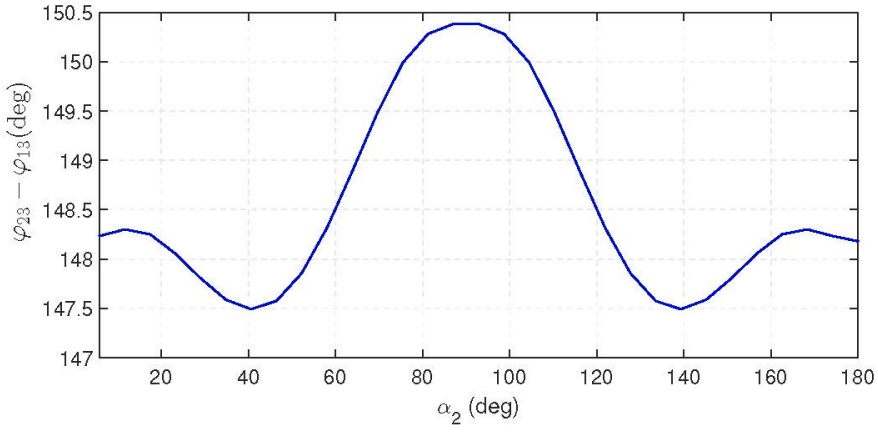

(c)

Fig. 6. (a) Single split ring. (b) Phase of $R_{\mathrm{RHCP}, \mathrm{RHCP}}$ and $R_{\mathrm{LHCP}, \mathrm{LHCP}}$ for the reflectarray element of (a) as a function of the rotation angle of the ring $\alpha_{2}$. Convergence is studied with respect to the number of basis functions $(q=1, \ldots, 7)$ used in the two arcs of the split rings. Our results obtained with the spectral domain MoM are compared with results obtained with $\mathrm{CST}^{\circledR}$. (c) Variation of the length of the arcs of the split ring with the rotation angle $\alpha_{2}$ to ensure a phase difference of $180^{\circ}$ in the reflection coefficients of the two orthogonal linear components of the $\mathrm{CP}$ impinging waves. Parameters: $a=b=5 \mathrm{~mm} ; \rho_{13}=1.85 \mathrm{~mm} ; \rho_{23}=2.05 \mathrm{~mm} ; h_{1}=0.787 \mathrm{~mm}, \varepsilon_{r, 1}=2.2$, $\tan \delta_{1}=0.0009 ; h_{2}=0 \mathrm{~mm} ; \theta_{\mathrm{inc}}=30^{\circ}, \varphi_{\mathrm{inc}}=0^{\circ} ; f=19.95 \mathrm{GHz}$.

between the reflection coefficient of the component of the electric field along the mirror symmetry axis of the split ring and the reflection coefficient of its orthogonal component [2], [3]. To ensure this phase difference is maintained, the length of the ares in the reflectarray element has to be slightly adjusted as $\alpha_{2}$ is varied [6]. This adjustment of the length of the arcs as a function of $\alpha_{2}$ is shown in Fig. 6(c). In Fig. 6(b) we show the convergence of $\angle R_{\mathrm{RHCP}, \mathrm{RHCP}}$ and $\angle R_{\mathrm{LHCP}, \mathrm{LHCP}}$ as a function of the number of basis functions $N_{b}$ used in the two arcs of the split rings. It can be seen that just three basis functions per arc suffice to achieve convergence (in fact, the results obtained with the three basis functions corresponding 
to $q=1,2,3$ in (21) overlap those obtained when $N_{b}=7$ and $1 \leq q \leq 7$ ), which means that only $6 \times 6 \mathrm{MoM}$ matrices have to be inverted when analyzing the multilayered periodic structure containing single split rings in the unit cell. In order to validate the results obtained with the spectral MoM software described in Section II, these results are compared with results provided by the commercial software $\operatorname{CST}^{\circledR}$. Excellent agreement is found between both sets of results.

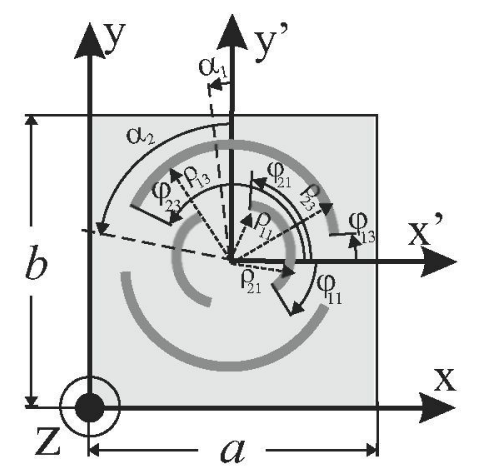

(a)

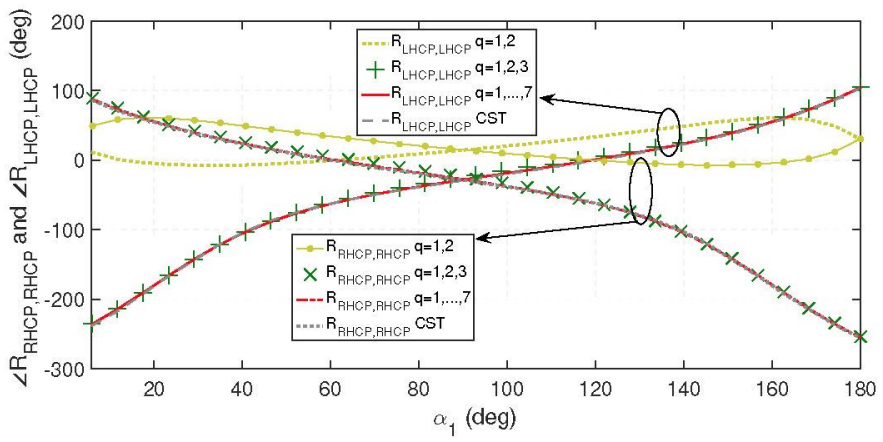

(b)

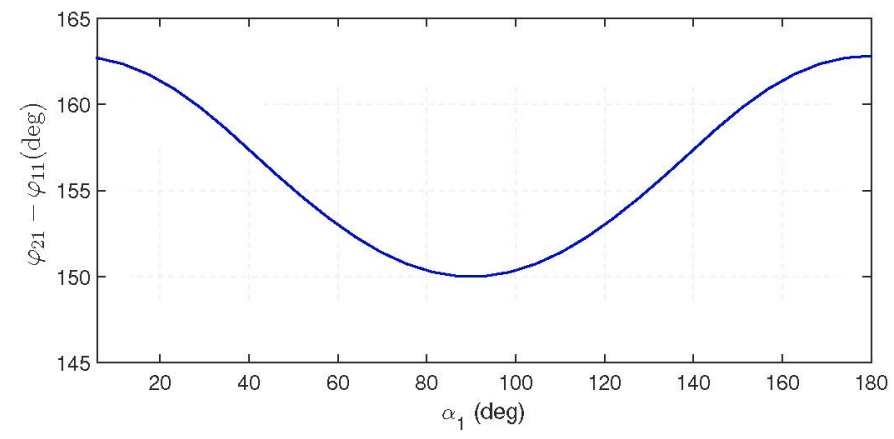

(c)

Fig. 7. (a) Two concentric split rings. (b) Phase of $R_{\mathrm{RHCP}, \mathrm{RHCP}}$ and $R_{\mathrm{LHCP}, \mathrm{LHCP}}$ for the reflectarray element of (a) as a function of the rotation angle of the inner ring $\alpha_{1}$. Convergence is studied with respect to the number of basis functions $(q=1, \ldots, 7)$ used in the four arcs of the two split rings. Our results obtained with the spectral domain MoM are compared with results obtained with $\operatorname{CST}^{\mathrm{B}}$. (c) Variation of the length of the ares of the inner ring with the rotation angle $\alpha_{1}$ to ensure a phase difference of $180^{\circ}$ in the reflection coefficients of the two orthogonal linear components of the CP impinging waves. Parameters: $a=b=5 \mathrm{~mm} ; \rho_{13}=1.85 \mathrm{~mm} ; \rho_{23}=2.05 \mathrm{~mm}$; $\varphi_{13}=-75.2^{\circ} \mathrm{mm} ; \varphi_{23}=75.2^{\circ} \mathrm{mm} ; \alpha_{2}=0^{\circ} ; \rho_{11}=1.20 \mathrm{~mm} ; \rho_{21}=1.40 \mathrm{~mm}$; $h_{1}=0.787 \mathrm{~mm}, \varepsilon_{r, 1}=2.2, \tan \delta_{1}=0.0009 ; h_{2}=0 \mathrm{~mm} ; \theta_{\text {inc }}=30^{\circ}, \varphi_{\text {inc }}=0^{\circ}$; $f=29.75 \mathrm{GHz}$.

In Fig. 7(b) we plot the phase curves for a dual-frequency $\mathrm{CP}$ reflectarray antenna made of two concentric split rings (see Fig.7(a)). While the outer rings are intended to adjust the phase
TABLE I

CPU TTMES (SECONDS) REQUIRED FOR THE GENERATION OF 31 POINTS IN THE CURVES of Figs.6(B) and 7(B). The CPU times are For our MoM software and CST ${ }^{\circledR}$.

\begin{tabular}{c|c|c}
\hline Figure & $\mathrm{T}_{\mathrm{CPU}}^{\mathrm{MoM}}$ & $\mathrm{T}_{\mathrm{CPU}}^{\mathrm{CST}}$ \\
\hline \hline Fig. 6(b) & 89.8 & 2383 \\
\hline \hline Fig. 7(b) & 152.9 & 2486 \\
\hline
\end{tabular}

TABLE II

CPU TIMES (SECONDS) CONTRIBUTIONS (COMPUTATION OF HANKEL TRANSFORMS, INTERPOLATION OF HANKEL TRANSFORMS, CALCULATION OF 2-D FOURIER TRANSFORMS OF THE BASIS FUNCTIONS AND EVALUATION OF MATRIX ENTRIES) TO THE TOTAL CPL TIME REQUIRED BY OUR MOM SOFTWARE FOR THE GENERATION OF ONE POINT IN FIGS, 6(B) AND 7(B).

\begin{tabular}{c|c|c|c|c|c}
\hline Figure & $\mathrm{T}_{\mathrm{HT}}$ & $\mathrm{T}_{\mathrm{INT}}$ & $\mathrm{T}_{2 \mathrm{DFT}}$ & $\mathrm{T}_{\mathrm{ME}}$ & $\mathrm{T}_{\mathrm{TOTAL}}^{\mathrm{MoM}}$ \\
\hline \hline Fig. 6(b) & 0.398 & 0.0005 & 2.868 & 0.007 & 3.290 \\
\hline \hline Fig. 7(b) & 0.570 & 0.0008 & 4.871 & 0.020 & 5.477 \\
\hline
\end{tabular}

at $19.95 \mathrm{GHz}$, the inner rings are intended to adjust the phase at $29.75 \mathrm{GHz}$ as in [6]. In Fig. 7(b) we plot the curves for phase adjustment at $29.75 \mathrm{GHz}$. Since this phase adjustment is controlled by the inner rings, in the application of the VRT $\angle R_{\mathrm{RHCP}, \mathrm{RHCP}}$ and $\angle R_{\mathrm{LHCP}, \mathrm{LHCP}}$ are varied by rotating the mirror symmetry angle of the inner split ring, $\alpha_{1}$, while keeping fixed the mirror symmetry axis of the outer ring $\alpha_{2}$. Note that whereas $\angle R_{\mathrm{RHCP}, \mathrm{RHCP}}$ and $\angle R_{\mathrm{LHCP}, \mathrm{LHCP}}$ are linear functions of $\alpha_{2}$ in Fig. $6(\mathrm{~b}), \angle R_{\mathrm{RHCP}, \mathrm{RHCP}}$ and $\angle R_{\mathrm{LHCP}, \mathrm{LHCP}}$ are not any longer linear functions of $\alpha_{1}$ in Fig. 7(b). This is due to the fact that whereas in Fig. 6(b) the whole reflectarray element is rotated for phase adjustment, in Fig. 7(b) only the inner part of the element is rotated for phase adjustment, and therefore the proportionality between phase and rotation angle proven in [2] is lost. Fortunately, the rotation of $\alpha_{1}$ in Fig. 7(b) provides enough phase range for reflectarray design. In Fig. 7(c) we plot the adjustments in the length of the inner arcs that are needed to keep a phase difference of $180^{\circ}$ between the reflection coefficients of the two orthogonal components of the electric field of the impinging $\mathrm{CP}$ waves. These adjustments are larger than in the case of Fig. 6(c), which is attributed to the fact that the outer arcs of the element are not modified during the process of phase adjustment. As in Fig. 6(b), in Fig. 7(b) convergence of the spectral domain MoM software is achieved with just three basis functions per arc (which requires the inversion of $12 \times 12 \mathrm{MoM}$ matrices), and excellent agreement is found between the spectral domain MoM software and CST ${ }^{\circledR}$.

We have computed the CPU time required by $\mathrm{CST}^{\circledR}$ to generate 31 points of the curves of Figs. $6(\mathrm{~b})$ and $7(\mathrm{~b}), \mathrm{T}_{\mathrm{CPU}}^{\mathrm{CST}}$, and the CPU time required by the spectral domain MoM software when 3 basis functions per arc are employed, $\mathrm{T}_{\mathrm{CPU}}^{\mathrm{MoM}}$. When running the spectral MoM software, we have used the interpolated versions of $\tilde{f}_{l}^{\mathrm{i}}\left(k_{\rho}\right)$, and we have included in $\mathrm{T}_{\mathrm{CPU}}^{\mathrm{MoM}}$ the CPU time required to compute $k_{\rho}^{\text {th }}(i)$ and interpolate the 
functions $\tilde{f}_{l}^{i}\left(k_{\rho}\right)$. The results obtained for $\mathrm{T}_{\mathrm{CPI}}^{\mathrm{CST}}$ and $\mathrm{T}_{\mathrm{CPU}}^{\mathrm{MoM}}$ are shown in Table I. These CPU times have been obtained in a laptop computer with processor Intel Core i7-6700HQ at $2.6 \mathrm{GHz}$ with four cores and $32 \mathrm{~GB}$ of RAM memory. The MoM code has been written in FORTRAN language. According to the results of Table I, the spectral MoM software is typically 27 times faster than $\operatorname{CST}^{\circledR}$ in the analysis of the periodic structures studied in Fig. 6(b), and around 16 times faster than $\mathrm{CST}^{\circledR 8}$ in the analysis of the periodic structures of Fig. 7(b). When the spectral domain MoM described in Section II is applied to the analysis of one single periodic structure containing split rings, there are different numerical steps having different CPU time contributions to the total CPU time required by MoM, $\mathrm{T}_{\mathrm{TOTAL}}^{\mathrm{MoM}}$. These $\mathrm{CPU}$ time contributions include the CPU time required to compute the Hankel transforms $\tilde{f}_{l}^{i}\left(k_{\rho}\right)$ that are needed for the interpolations by means of (24), $\mathrm{T}_{\mathrm{HT}}$, the CPU time required for the interpolations of the Hankel transforms by means of Chebyshev polynomials, $\mathrm{T}_{\mathrm{INT}}$, the CPU time required for the evaluation of the 2-D Fourier transforms of the basis functions by means of (18), $\mathrm{T}_{2 \mathrm{DFT}}$, and the CPU time required for the determination of the MoM matrix entries by means of (6), $\mathrm{T}_{\mathrm{ME}}$. Table II shows these different CPU time contributions to $\mathrm{T}_{\text {TOTAL }}^{\mathrm{M} M}$ (the CPU times have been obtained with the same computer used for Table I). In particular, the CPU times presented in Table II are for the periodic structure analyzed in Fig. 6(b) when $\alpha_{2}=92.9^{\circ}$ $\left(\varphi_{23}-\varphi_{13}=150.4^{\circ}\right.$ in Fig. 6(c)), and for the periodic structure analyzed in Fig. $7(\mathrm{~b})$ when $\alpha_{1}=92.9^{\circ}\left(\varphi_{21}-\varphi_{11}=150.0^{\circ}\right.$ in Fig. 7(c)). Note that the most important contribution to the total MoM CPU time is $\mathrm{T}_{2 \mathrm{DFT}}$, which is around $88 \%$ of $\mathrm{T}_{\text {TOTAL }}^{\mathrm{MOM}}$. This justifies the efforts carried out in Section II to compute the Hankel transforms $\tilde{f}_{l}^{i}\left(k_{\rho}\right)$ appearing in (18) in an efficient way. If we had not optimized the computation of $\tilde{f}_{l}^{i}\left(k_{\rho}\right)$, the method presented in this paper would not have been competitive by comparison with commercial software. Once the functions $\widetilde{\mathbf{J}}_{l q}^{\mathrm{d}}\left(k_{x m}, k_{y n}\right)$ of (18) have all been computed, the CPU times required to compute the double summations of (6) and to solve the system of equations of (5) are negligible (note that the maximum size of the MoM matrices to be inverted is $12 \times 12$ ). The next important contribution to the total MoM CPU time is $\mathrm{T}_{\mathrm{HT}}$, which represents around $11 \%$ of $\mathrm{T}_{\mathrm{TOTAL}}^{\mathrm{MoM}}$. Table II shows the contribution of the time required for the interpolation of the Hankel transforms to $\mathrm{T}_{\mathrm{TOTAL}}^{\mathrm{MOM}}$ is negligible. At this point, we should remember that the computation and interpolation of the Hankel tranforms has to be carried out only once in the design of a reflectarray antenna where the inner and outer radii of the rings $\rho_{1 l}$ and $\rho_{2 l}(l=1, \ldots, L)$ remain constant in all the elements of the antenna. Therefore, we can neglect the contribution of $\mathrm{T}_{\mathrm{HT}}$ to the CPU time required in the design of a whole reflectarray antenna. In our study of the computational performance of the spectral domain MoM software as a function of the different input variables involved, we have finally analyzed the computational complexity of $\mathrm{T}_{\mathrm{TOTAL}}^{\mathrm{MoM}}$ as a function of the number of quadrature points used in (24), $N_{\mathrm{qp}}$, as a function of the number of terms retained in infinite series of (18), $i_{\max }$, and as a function of the number of basis functions used in the application of MoM, $N_{b}$. We have found that the effect of $N_{\mathrm{qp}}$ on $\mathrm{T}_{\mathrm{TOTAL}}^{\mathrm{MMM}}$ is irrelevant for $3 \leq N_{\text {qp }} \leq 15$, that the spectral MoM is roughly of complexity $O\left(\left(i_{\max }\right)^{2}\right)$ for $48 \leq i_{\max } \leq 200$, and that the spectral MoM is roughly of complexity $O\left(N_{\mathrm{b}}\right)$ for $1 \leq N_{\mathrm{b}} \leq 7$.

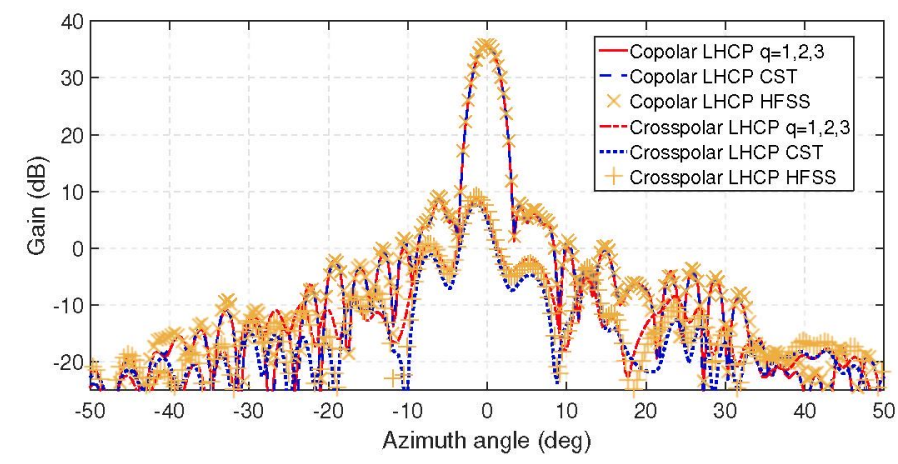

(a)

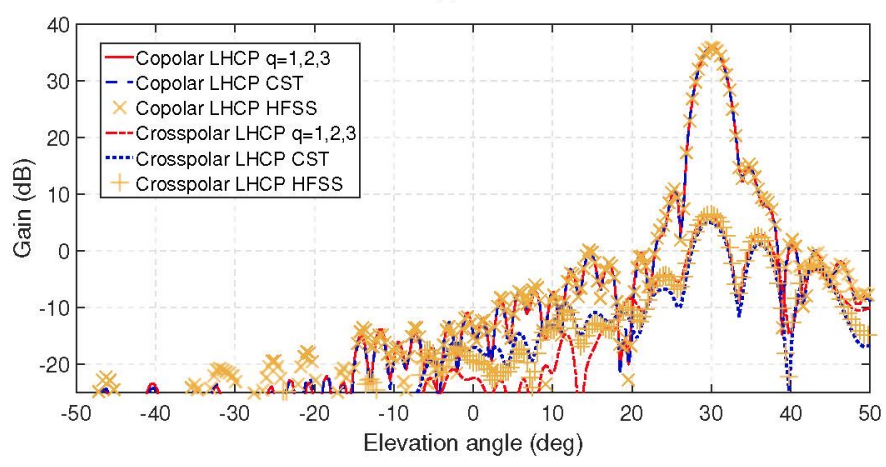

(b)

Fig. 8. Radiation patterns in (a) the azimuth plane and (b) the elevation plane for a dual-frequency $\mathrm{CP}$ reflectarray based on an element with two concentric split rings. Plots are presented for the analysis of the reflectarray under the local periodicity assumption with both our spectral domain MoM software (solid and dash-dotted lines), CST ${ }^{\mathbb{B}}$ (dashed and dotted lines) and $\operatorname{HFSS}^{\mathbb{B}}(\times$ and +). The results plotted are for LHCP radiation at $19.95 \mathrm{GHz}$.

Based on the curves of Figs. 6(b), 6(c), 7(b), and 7(c), a dual-frequency pencil beam CP reflectarray antenna has been designed. The antenna radiates LHCP at $19.95 \mathrm{GHz}$ and RHCP at $29.75 \mathrm{GHz}$ as in the case of the antenna designed in [6]. The designed antenna is circular and consists of 5024 elements arranged in a $80 \times 80$ grid with cell size $5 \mathrm{~mm} \times 5 \mathrm{~mm}$ (the diameter of the antenna is $400 \mathrm{~mm}$ ). The antenna is intended to produce a focused beam in the direction $\theta_{b}=30^{\circ}$ and $\varphi_{b}=0^{\circ}$ (see [25, Fig. 5.a]). It is illuminated by a corrugated circular feed-horn with its phase center located at the coordinates $x=-150 \mathrm{~mm}, y=0 \mathrm{~mm}, z=259.8 \mathrm{~mm}$ with respect to a coordinate system with origin at the center of the reflectarray (see [25, Fig. 5.a]). The horn is assumed to radiate LHCP waves at $19.95 \mathrm{GHz}$ and RHCP waves at $29.75 \mathrm{GHz}$. The radiation pattern of the horn is modelled as a function $\cos ^{7}(\theta)$, which provides an illumination level at the reflectarray edges $12 \mathrm{~dB}$ below the maximum. The antenna elements are dual concentric split rings as those shown in Fig. 7(a). In the design of the antenna at $19.95 \mathrm{GHz}$, the dimensions and orientations of the outer rings have been adjusted in accordance with Figs. 6(b) and 6(c), while assuming the inner rings were not present. However, when the antenna has been designed at $29.75 \mathrm{GHz}$, both the outer and the inner rings have been taken 


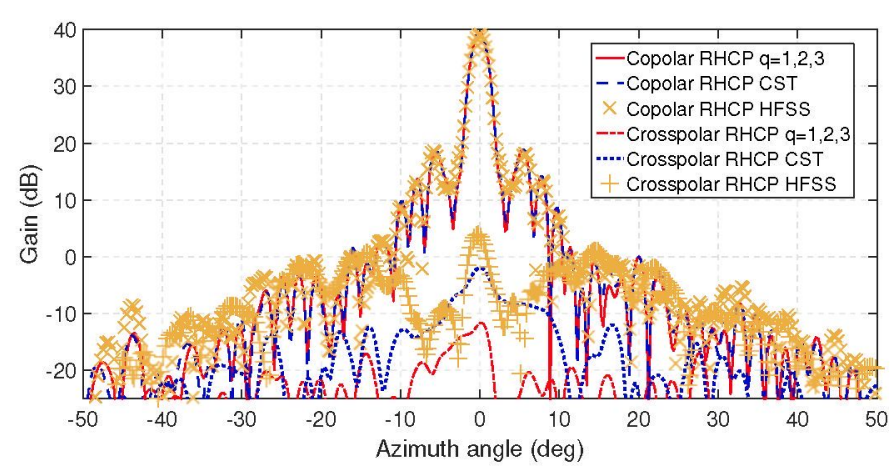

(a)

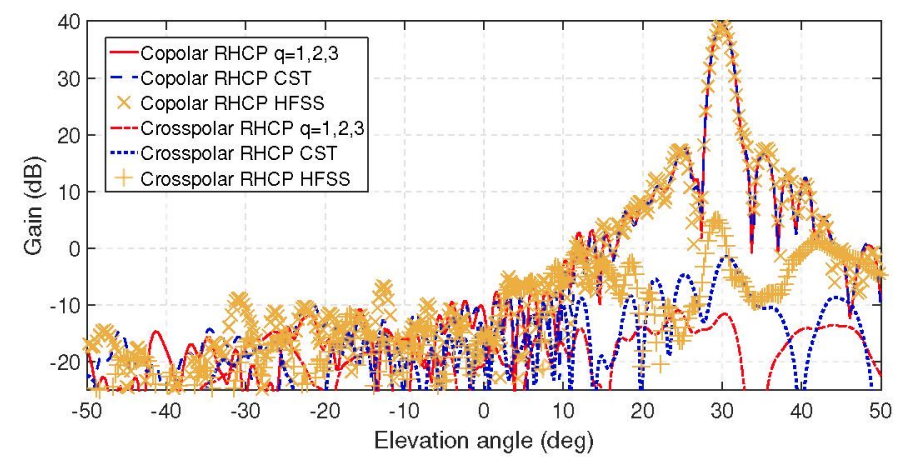

(b)

Fig. 9. Radiation patterns in (a) the azimuth plane and (b) the elevation plane for a dual-frequency CP reflectarray based on an element with two concentric split rings. Plots are presented for the analysis of the reflectarray under the local periodicity assumption with both our spectral domain MoM software (solid and dash-dotted lines), CST $^{\circledR}$ (dashed and dotted lines) and HFSS ${ }^{\circledR}(\times$ and + ). The results plotted are for RHCP radiation at $29.75 \mathrm{GHz}$.

into account. The dimensions and orientations of the outer rings have been fixed at the values obtained for the design at $19.95 \mathrm{GHz}$, and the dimensions and orientations of the inner rings have been adjusted in accordance with Figs. 7(b) and 7(c). The design of the antenna has been carried out by means of the spectral domain MoM software described in Section II under the local periodicity condition, while using the exact incidence angles in each reflectarray element. Once the reflectarray antenna has been designed, it has been analyzed with the spectral domain MoM software (using three basis functions per arc with $q=1,2,3$ in (21)), and with the two commercial softwares CST ${ }^{\circledR}$ and HFSS ${ }^{\circledR}$, under the local periodicity assumption. In the analysis, the exact incidence angles has been considered in each reflectarray element. Figs. 8 and 9 show the radiation patterns obtained in the azimuth and elevation planes at $19.95 \mathrm{GHz}$ and $29.75 \mathrm{GHz}$ respectively. The resulting gain is $35.88 \mathrm{dBi}$ at $19.95 \mathrm{GHz}$ and $39.42 \mathrm{dBi}$ at $29.75 \mathrm{GHz}$. And the antenna efficiency is of $56 \%$ at 19.95 GHz and $57 \%$ at 29.75 GHz. In Fig. 8 the agreement between our MoM results, CST ${ }^{\circledR}$ and HFSS ${ }^{\circledR}$ is excellent. In Fig. 9 the agreement is excellent for the copolar component of the radiation patterns, but it is not so good for the cross-polar component. In fact, discrepancies exist not only between our MoM results and those of both $\operatorname{CST}^{\circledR}$ and $\mathrm{HFSS}^{\circledR}$, but also between CST $^{\circledR}$ and HFSS ${ }^{\circledR}$. This is attributed to the difficulties encountered to correctly reproduce cross-polarization levels
TABLE III

CPU TIMES (HOURS) REQUIRED FOR THE ANALYSIS OF THE DUAL-FREQUENCY reflectarray antenNa of Figs. 8 and 9 at $19.95 \mathrm{GHz}$ and $29.75 \mathrm{GHz}$. The CPU TIMES ARE FOR OUR MOM SOFTWARE, CST ${ }^{\circledR}$ AND HFSS ${ }^{\circledR}$.

\begin{tabular}{l|l|l}
\hline $\mathrm{T}_{\mathrm{CPU}}^{\mathrm{MoM}}$ & $\mathrm{T}_{\mathrm{CPU}}^{\mathrm{CST}}$ & $\mathrm{T}_{\mathrm{CPU}}^{\mathrm{HFSS}}$ \\
\hline \hline 14.08 & 392.6 & 363.0 \\
\hline
\end{tabular}

which are between 40 and $50 \mathrm{~dB}$ below the maximum of the copolar radiation pattern. Probably, a better agreement for the cross-polarization in Figs. 9(a) and 9(b) could have been achieved if we had used denser meshes in CST $^{\circledR}$ and HFSS ${ }^{\circledR}$ at the expense of a higher CPU time consumption. Note it is a logical result that the cross-polarization at $19.95 \mathrm{GHz}$ (around $30 \mathrm{~dB}$ below the maximum) is larger than the crosspolarization at $29.75 \mathrm{GHz}$ (between 40 and $50 \mathrm{~dB}$ below the maximum) since the antenna analyzed is made of elements with two split rings, and whereas the inner split rings were not considered in the design at $19.95 \mathrm{GHz}$, both split rings were considered in the design at $29.75 \mathrm{GHz}$. Table III shows a comparison of the total CPU times required for the analysis of the antenna with our MoM software, CST ${ }^{\circledR}$ and HFSS ${ }^{\circledR}$ at both frequencies $19.95 \mathrm{GHz}$ and $29.75 \mathrm{GHz}$. The CPU times are measured in the laptop computer used to generate the results of Tables I and II. Please note that our MoM software is around 28 times faster than CST $^{\circledR}$, and 26 times faster than HFSS ${ }^{\circledR}$. These important CPU time gains shows the advantage of using the in-house software proposed in this paper for the analysis and design of CP reflectarray antennas made of split rings.

\section{Conclusion}

In this paper the authors describe an efficient implementation of the spectral domain MoM for the analysis of multilayered periodic structures containing split rings in the unit cell. The spectral domain MoM software is subsequently used in the analysis and design of CP reflectarray antennas made of concentric split rings under the local periodicity assumption. When applying MoM, basis functions with edge singularities are used in the approximation of the current density on the arcs of the rings, and it is found that three basis functions per arc suffice to obtain convergence in the application of MoM when the width of the arcs is much smaller than their length, which is a condition usually fulfilled in the elements of reflectarray antennas. Since the 2-D Fourier transform of the basis functions with edge singularities cannot be obtained in closed form, these Fourier transforms are expressed as infinite series of Hankel transforms. The Hankel transforms are numerically computed by means of low order Gauss-Chebyshev quadrature rules. Also, analytical criteria are obtained for the number of terms to be retained in the infinite series as a function of the values of the spectral variables. Finally, Gauss-Chebyshev interpolations are invoked to accelerate the computation of the Hankel transforms. The spectral domain MoM software implemented has been used to analyze multilayered periodic structures containing split rings in the unit cell, and to analyze reflectarray antennas made 
of split rings under the local periodicity assumption. In both cases, the spectral domain MoM results have been compared with results provided by the commercial softwares $\mathrm{CST}^{\circledR}$ and HFSS $^{\circledR}$. Good agreement has been found between both sets of results, the spectral domain MoM software being around 27 times faster than $\mathrm{CST}^{\circledR}$ and $\mathrm{HFSS}^{\circledR}$.

\section{APPENDix A}

When the functions $g_{l q}\left(\varphi^{\prime}\right)$ of (21) are introduced in (15) and $q$ is odd, the resulting expressions obtained for $\widetilde{\mathbf{g}}_{l q}^{i}$ can be written as

$$
\widetilde{\mathbf{g}}_{l q}^{i}=(-1)^{\frac{q-1}{2}}\left[-\mathbf{j} b_{l q, \mathrm{x}}^{i} \hat{\mathbf{x}}+b_{l q, \mathrm{y}}^{i} \hat{\mathbf{y}}\right]
$$

where

$$
\begin{aligned}
b_{l q, \mathrm{x}}^{i}= & \frac{q}{4} \mathrm{e}^{-\mathrm{ji}\left(\frac{\varphi_{2 l} \varphi_{1 l}}{2}\right)} \\
& \times\left[\frac{\mathrm{e}^{-\mathrm{j}\left(\frac{\varphi_{2 l}+\varphi_{1 l}}{2}\right)}}{i+1} J_{q}\left(\frac{(i+1)\left(\varphi_{2 l}-\varphi_{1 l}\right)}{2}\right)\right. \\
& \left.-\frac{\mathrm{e}^{+\mathrm{j}\left(\frac{\varphi_{2 l}+\varphi_{1 l}}{2}\right)}}{i-1} J_{q}\left(\frac{(i-1)\left(\varphi_{2 l}-\varphi_{1 l}\right)}{2}\right)\right] \\
b_{l q, \mathrm{y}}^{i}= & \left.\frac{q}{4} \mathrm{e}^{-\mathrm{j} i\left(\frac{\varphi_{2 l} \varphi_{1 l}}{2}\right)}\right) \\
& \times\left[\frac{\mathrm{e}^{-\mathrm{j}\left(\frac{\varphi_{2 l}+\varphi_{1 l}}{2}\right)}}{i+1} J_{q}\left(\frac{(i+1)\left(\varphi_{2 l}-\varphi_{1 l}\right)}{2}\right)\right. \\
& \left.+\frac{\mathrm{e}^{+\mathrm{j}\left(\frac{\varphi_{2 l}+\varphi_{1 l}}{2}\right)}}{i-1} J_{q}\left(\frac{(i-1)\left(\varphi_{2 l}-\varphi_{1 l}\right)}{2}\right)\right]
\end{aligned}
$$
by

In case $q$ is even, the resulting expressions for $\widetilde{\mathbf{g}}_{l q}^{i}$ are given

$$
\widetilde{\mathbf{g}}_{l q}^{i}=(-1)^{\frac{q}{2}}\left[b_{l q, \mathrm{x}}^{i} \hat{\mathbf{x}}+\mathbf{j} b_{l q, \mathrm{y}}^{i} \hat{\mathbf{y}}\right]
$$

\section{REFERENCES}

[1] J. Huang and J. A. Encinar, Reflectarray antennas. Piscataway, NJ/New York: IEEE Press/Wiley, 2008.

[2] J. Huang and R. Pogorzelski, "A Ka-band microstrip reflectarray with elements having variable rotation angles," IEEE Trans. Antennas Propagat., vol. 46, no. 5, pp. 650-656, May 1998.

[3] A. E. Martyniyuk, J. I. Martinez Lopez, and N. I. Martynyuk, "Spiraphase-type reflectarrays based on loaded ring slot resonators," IEEE Trans. Antennas Propagat., vol. 52, no. 1, pp. 142-153, Jan. 2004.

[4] C. Han and K. Chang, "Ka-band reflectarray using ring elements," Electron. Lett., vol. 39, no. 6, pp. 491-493, March 2003.

[5] C. Han, C. Rodenbeck, J. Huang, and K. Chang, "A C/Ka dual frequency dual layer circularly polarized reflectarray antenna with microstrip ring elements", vol. 52, no. 11, Nov. 2004, pp. 2871-2876.

[6] T. Smith, U. Gothelf, O. S. Kim, and O. Brenbjerg, "Design, manufacturing and testing of a $20 / 30-\mathrm{GHz}$ dual-band circularly polarized reflectarray antenna," IEEE Trans. Antennas Wireless Propagat. Lett., vol. 12, pp. $1480-1483,2013$

[7] T. Smith, U. Gothelf, O. S. Kim, and O. Brenbjerg, "An FSS backed 20/30 GHz circularly polarized reflectarray for a shared aperture L- and Ka-band satellite communication antenna," IEEE Trans. Antennas Propagat., vol. 62, no. 2, pp. 661-668, Feb. 2014.

[8] M.-Y. Zhao, G.-Q. Zhang, X. Lei, J.-M. Wu, and J.-Y. Shang, "Design of new single-layer multiple-resonance broadband circularly polarized reflectarrays," IEEE Trans. Antennas Wireless Propagat. Lett., vol. 12, pp. 356-359, 2013.
[9] X. Zhang, F. Yang, S. Xu, and M. Li, "A wideband reflectarray design using novel phasing rings," Proc. ICEAA 2017, Verona, Italy, September, 2017, pp. 178-180.

[10] D.M. Pozar, T.A. Metzler, "Analysis of a reflectarray antenna using microstrip patches of variable size," Electron. Lett., vol. 29, no. 8, pp. 657-658, April 1993.

[11] D.M. Pozar, S.D. Targonski, H.D. Syrigos, "Design of millimeter wave microstrip reflectarray," IEEE Trans. Antennas Propagat., vol. 45, no. 2, pp. 287-296, February 1997.

[12] S. G. Pintzos and R. Pregla, "A simple method for computing the resonant frequencies of microstrip ring resonators," IEEE Trans. Microwave Theory Tech., vol. MTT-26, pp. 809-813, no. 10, Oct. 1978.

[13] S. M. Ali, W. C. Chew, and J. A. Kong, "Vector Hankel transform analysis of annular ring microstrip antennas," IEEE Trans. Antennas Propagat., vol. AP-30, no. 4, pp. 637-644, July 1982.

[14] Z. Fan and K.-F. Lee, "Hankel transform domain analysis of dualfrequency stacked circular-disk and annular-ring microstrip antennas," IEEE Trans. Antennas Propagat., vol. 39, no. 6, pp. 867-870, June 1991.

[15] F. Tefiku and E. Yamashita, "An efficient method for the determination of the resonant frequencies of shielded circular disk and ring resonators," IEEE Trans. Microwave Theory Tech., vol. 41, pp. 343-346, no. 2, Feb. 1993.

[16] J. T. Kuo, "Vector finite Hankel transform analysis of shielded single and coupled microstrip ring structures," IEEE Trans. Antennas Propagat., vol. 47, no. 11, pp. 2161-2164, Nov. 1999.

[17] E. Khodapanah and S. Nikmehr, "On the use of subsectional spline basis functions for efficient calculation of the RCS of a microstrip array of concentric anular rings over a wide frequency band," IEEE Trans. on Antennas and Propag., vol. 57, no. 3, pp. 825-830, March 2009.

[18] I. Wolff and V. K. Tripathi, "The microstrip open-ring resonator," IEEE Trans. Microwave Theory Tech., vol. MTT-32, pp. 102-107, no. 1, Jan. 1984.

[19] G. Vecchi, P. Pirinoli, and M. Orefice, "On the use of cavity mode as basis functions in the full wave analysis of printed antennas," IEEE Trans. on Antennas and Propag., vol. 46, pp. 589-594, no. 4, April 1998.

[20] R. Florencio, R. R. Boix, J. A. Encinar, and G. Toso, "Optimized periodic MoM for the analysis and design of dual polarization multilayered reflectarray antennas made of dipoles," IEEE Trans. on Antennas and Propag., vol. 65, pp. 3623-3637, no. 7, July 2017.

[21] F. Mesa, R. Marqués, and M. Horno, "A general algorithm for computing the bidimensional spectral Green's dyads in multilayered complex bianisotropic media: the equivalent boundary method," IEEE Trans. Microwave Theory Tech., vol. 39, pp. 1640-1649, no. 9, Sept. 1991.

[22] R. Mittra, C. H. Chan, and T. Cwik, "Techniques for analyzing frequency selective surfaces-A review", Proc. IEEE. vol. 76, no. 12, pp. 1593-1615, Dec. 1988.

[23] M. Abramowitz and I. Stegun, Handbook of Mathematical Functions, New York: Dover Publications, 9th Edition, 1970.

[24] A. G. Polimeridis and J. R. Mosig, "Evaluation of weakly singular integrals via generalized cartesian product rules based on the double exponential formula," IEEE Trans. Antennas Propagat., vol. 58, pp. 19801988, no. 6, June 2010.

[25] R. Florencio, J. A. Encinar, R. R. Boix, and G. Pérez Palomino, "Dualpolarization reflectarray made of cells with two orthogonal sets of parallel dipoles for bandwidth and cross-polarization improvement," IET Microw. Antennas Propag., Vol. 8, Iss. 15, pp. 1389-1397, 2014.

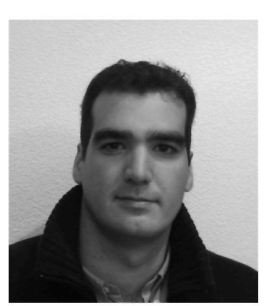

Rafael Florencio received the "Licenciado" degree in Physics from the University of Seville, Spain, in 2008, the "Master in Communications Technology and Systems" from the Polytechnic University of Madrid, Spain, in 2010, and the "Ph. D. in Telecommunications Engineering" from the Polytechnic University of Madrid, Spain, in 2016. His current research interests are focused on the efficient design of dual-polarization dual-frequency reflectarray antennas for satellite applications.

Mr. Florencio was the recipient of a Scholarship financed by the "Junta de Andalucía" to carry out his Ph. D. thesis. 


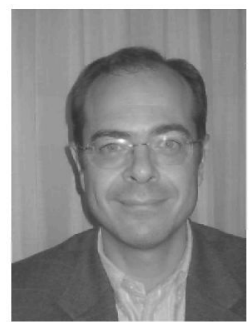

Rafael R. Boix (M'96) received the "Licenciado" and "Doctor" degrees in physics from the University of Seville, Spain, in 1985 and 1990 respectively.

Since 1986, he has been with the Electronics and Electromagnetism Department, University of Seville, where he became Tenured Professor in 2010. His current research interests are focused on the efficient numerical analysis of periodic planar multilayered structures with applications to the design of frequency selective surfaces and reflectarray antennas.

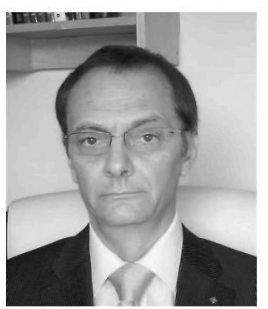

José A. Encinar (S'81-M'86-SM'09-FM'10) was born in Madrid, Spain. He received the Electrical Engineer and Ph.D. degrees, both from "Universidad Politécnica de Madrid (UPM)", in 1979 and 1985, respectively.

Since January 1980, he has been with the Applied Electromagnetism and Microwaves Group at UPM, as a Teaching and Research Assistant from 1980 to 1982, as an Assistant Professor from 1983 to 1986, and as Associate Professor from 1986 to 1991. From February to October of 1987 he stayed at Polytechnic University, Brooklyn, NY, as a Post-doctoral Fellow of the NATO Science Program. Since 1991 he has been a Professor of the Electromagnetism and Circuit Theory Department at UPM. He was with the Laboratory of Electromagnetics and Acoustics at "École Polytechnique Fédérale de Lausanne (EPFL)", Switzerland in 1996, and with the Institute of Electronics, Communication and Information Technology (ECIT), Queen's University Belfast, U.K. in 2006 and 2011, as a Visiting Professor. His research interests include numerical techniques for the analysis of multilayered periodic structures, design of frequency selective surfaces, printed arrays and reflectarrays. Prof. Encinar has published more than one hundred and fifty journal and conference papers, and he is holder of five patents on array and reflectarray antennas.

He was a co-recipient of the 2005 H. A. Wheeler Applications Prize Paper Award and the 2007 S. A. Schelkunoff Transactions Prize Paper Award, given by IEEE Antennas and Propagation Society. He has been a member of the Technical Programme Committee of several International Conferences (European Conference on Antennas and Propagation, ESA Antenna Workshops, and Loughborough Antennas \& Propagation Conference). He was appointed IEEE Fellow by the Antennas \& Propagation Society in 2010. 